\title{
Comparação das respostas do VEMP cervical e ocular em indivíduos com e sem doenças otoneurológicas
}

\author{
Comparison of cervical and ocular VEMP \\ responses in individuals with and without \\ otoneurological diseases
}

\author{
Comparación de respuestas VEMP \\ cervicales y oculares en individuos con y sin \\ enfermedades otoneurológicas
}

\author{
Marlon Bruno Nunes Ribeiro* (iD \\ Patricia Cotta Mancini*
}

\section{Resumo}

Introdução: a avaliação vestibular é realizada tradicionalmente por meio da prova calórica para avaliação do labirinto em indivíduos com tonturas, porém este exame não avalia toda a via vestibular. Os potenciais evocados miogênicos vestibulares (VEMP) consistem em um exame de rápida execução, considerado método de escolha para avaliação dos órgãos otolíticos e do nervo vestibular. Objetivo: analisar as respostas do exame VEMP cervical (cVEMP) e ocular (oVEMP) em indivíduos com doenças vestibulares e compará-las àquelas obtidas em indivíduos de mesma faixa etária e sexo sem queixas de tontura pregressas e atuais. Métodos: Estudo observacional, transversal e analítico. Os participantes foram divididos em dois grupos: grupo com tontura (GT), composto por indivíduos com diversas doenças vestibulares e o grupo sem queixa de tontura (GC). Ambos os grupos foram submetidos à meatoscopia, ao VEMP cervical e ocular. Resultados: A amostra foi composta por 45 indivíduos com a idade entre 23 e 68 anos, sendo 27 indivíduos do grupo GC e 18 do grupo GT. No exame cVEMP as latências, amplitudes, índice de assimetria e o índice de assimetria corrigido foram iguais no GT quando comparado com o GC. No exame oVEMP encontrou-se a amplitude esquerda maior e a latência N10 menor estatisticamente no grupo GT quando comparado ao grupo GC. Dentre as doenças do grupo GT os indivíduos com deiscência de canal superior tiveram valores maiores na amplitude esquerda, o que pode ter interferido nos resultados. Conclusão: Não foram evidenciadas diferenças nas respostas do cVEMP entre os grupos neste estudo. Encontrou-se aumento da amplitude esquerda e o valor menor da latência N10 direita no grupo com tontura na análise do exame oVEMP. Acredita-se que a heterogeneidade de

* Universidade Federal de Minas Gerais, Belo Horizonte, MG, Brasil.

Contribuição dos autores:

MBNR - idealizou o estudo, realizou a coleta de dados, participou da análise estatística, da redação e revisão do manuscrito. PCM - participou, na condição de orientadora, da idealização do estudo, análise estatística, redação e revisão do manuscrito.

E-mail para correspondência: Marlon Bruno Nunes Ribeiro - marlonfono16@gmail.com Recebido: $26 / 06 / 2019$

Aprovado: 30/06/2020 
doenças vestibulares no grupo com tontura e o reduzido número de participantes em ambos os grupos tenham contribuído para esse desfecho.

Palavras-chave: Orelha interna; Equilíbrio Postural; Tontura; Potencial Evocado Motor; Sáculo e Utrículo

\section{Abstract}

Introduction: The vestibular assessment is traditionally carried out with the caloric test to evaluate the labyrinth in individuals with dizziness. However, this examination does not evaluate the entire vestibular pathway. The vestibular evoked myogenic potentials (VEMP) are a quick test, considered a choice method to assess the otolith organs and the vestibular nerve. Purpose: To analyze the responses of the cervical (cVEMP) and ocular VEMP (oVEMP) examinations in individuals with vestibular diseases and compare them with the results obtained in individuals of the same age group and gender without previous and current complaints of dizziness. Methods: In this observational, cross-sectional, analytical study, the participants were divided into two groups: the dizziness group (DG), composed of individuals with several vestibular diseases, and the group without complaints of dizziness (CG). Both groups underwent meatoscopy, and cervical and ocular VEMP. Results: The sample comprised 45 individuals aged 23 to 68 years -27 individuals in the CG and 18 in the DG. In the cVEMP exam, the latencies, amplitudes, asymmetry index, and the corrected asymmetry index were the same in the GT when compared to the GC. In the cVEMP examination, the latencies, amplitudes, asymmetry index, and corrected asymmetry index were equal in the DG when compared with the CG. In the oVEMP examination, the left amplitude was statistically greater and the N10 latency, smaller in the DG when compared with the CG. Of the diseases in the DG, individuals with superior canal dehiscence had higher left amplitude values, which may have interfered with the results. Conclusion: There were no differences in cVEMP responses between the groups in this study. An increase in the left amplitude and the lower value in the right N10 latency were found in the DG in the analysis of the oVEMP examination. It is believed that the heterogeneity of vestibular diseases in the DG and the small number of participants in both groups have contributed to this outcome.

Keywords: Inner Ear; Postural Balance; Dizziness; Motor Evoked Potential; Saccule and Utricle

\section{Resumen}

Introducción: La evaluación vestibular se lleva a cabo tradicionalmente a través de la prueba calórica para evaluar el laberinto en individuos con mareos, sin embargo, este examen no evalúa toda la vía vestibular. Los potenciales miogénicos evocados vestibulares (VEMP) consisten en una prueba de funcionamiento rápido, considerada el método de elección para evaluar los órganos otolíticos y el nervio vestibular. Objetivo: Analizar las respuestas del examen VEMP cervical y ocular en individuos con enfermedades vestibulares y compararlas con las obtenidas en individuos del mismo grupo de edad y sexo sin quejas de mareos previas y actuales. Métodos:Estudio observacional, transversal y analítico. Los participantes se dividieron en dos grupos: grupo con mareos (GT), compuesto por individuos con varias enfermedades vestibulares y el grupo sin quejas de mareos (CG). Ambos grupos se sometieron a meatoscopia, VEMP cervical y ocular. Resultados: La muestra consistió en 45 individuos con edades comprendidas entre 23 y 68 años, 27 individuos del grupo GC y 18 del grupo GT. En el examen cVEMP, las latencias, amplitudes, índice de asimetría y el índice de asimetría corregido fueron los mismos en el GT en comparación con el CG. En el examen oVEMP, la amplitud izquierda fue mayor y la latencia N10 fue estadísticamente menor en el grupo GT en comparación con el grupo CG. Entre las enfermedades en el grupo GT, los individuos con dehiscencia del canal superior tenían valores más altos en la amplitud izquierda, lo que puede haber interferido con los resultados. Conclusión: No hubo diferencias en las respuestas de cVEMP entre los grupos en este estudio. Se encontró un aumento en la amplitud izquierda y un valor más bajo de la latencia N10 derecha en el grupo con mareos en el análisis del examen oVEMP. Se cree que la heterogeneidad de las enfermedades vestibulares en el grupo de mareos y el pequeño número de participantes en ambos grupos contribuyeron a este resultado.

Palabras clave: Oído Interno, Equilibrio Postural, Mareo, Potenciales Evocados Motores, Sáculo y Utrículo 


\section{Introdução}

O equilíbrio corporal é fundamental para as mudanças posturais que permitem a realização de movimentos com harmonia, conforto físico e mental, mantendo a postura ereta e evitando quedas ${ }^{1}$. Para que o equilíbrio seja mantido, faz-se necessária uma interação entre os sistemas vestibular, visual e proprioceptivo ${ }^{2}$. Uma alteração no sistema vestibular pode ser manifestada por meio da tontura ${ }^{2,3}$.

Os sintomas vestibulares mais frequentes, de acordo com a classificação dos sintomas vestibulares elaborada e publicada pela Bárány Society, manifestam-se como ilusão de movimento, rotatório (vertigem) ou não, ou sensação de orientação espacial alterada (tontura) $)^{3}$. Podem acometer indivíduos de qualquer faixa etária, sendo mais comuns entre a população adulta e idosa, com idade superior a 40 anos $^{3}$.

A avaliação do sistema vestibular consiste em provas de equilíbrio estático, dinâmico, provas oculares e a tradicional prova calórica, que avalia os canais semicirculares laterais. Existem exames complementares que auxiliam no topodiagnóstico da lesão, como os potenciais evocados miogênicos vestibulares, a posturografia, o Video Head Impulse Test (v-HIT), a prova rotatória pendular decrescente e os exames de imagem ${ }^{4}$.

Entre estes exames otoneurológicos complementares, os potenciais evocados miogênicos vestibulares (VEMP) são estudados desde 1960, porém são utilizados para avaliar os reflexos sáculo-cólicos apenas a partir de $1992^{5,6}$. O VEMP cervical e ocular atualmente são testes de escolha para avaliação dos órgãos otolíticos e do nervo vestibular $^{5,6}$.

O VEMP cervical (cVEMP) é um exame objetivo, não invasivo, rápido, de fácil execução, de relativo baixo custo e que não traz desconforto ao paciente. Consiste em uma resposta miogênica, de curta latência, captada na musculatura cervical, após um estímulo sonoro de alta intensidade que estimula o sáculo e, consequentemente, a divisão inferior do nervo vestibular ${ }^{5,6,7}$. O cVEMP consiste em uma manifestação do reflexo vestíbulo-cólico. É uma resposta inibitória da contração do músculo esternocleidomastóideo, que gera um potencial bifásico ${ }^{5,6,7}$.

O VEMP ocular (oVEMP) é também uma resposta miogênica, de curta latência, evocada por estímulo sonoro de alta intensidade, que ativa o utrículo e, consequentemente, o nervo vestibular superior $^{8,9}$. Constitui uma manifestação do reflexo vestíbulo-ocular e apresenta-se como potencial bifásico, contralateral ao estímulo9.

Os parâmetros avaliados no VEMP são presença do potencial bifásico, latências, interamplitudes ou amplitudes pico-a-pico, e índice de assimetria das respostas. O cálculo do índice de assimetria é de grande importância, já que a interamplitude da onda obtida no VEMP varia conforme a intensidade da força de contração muscular realizada, que pode variar entre indivíduos ${ }^{8,9}$. A correção da interamplitude de acordo com a força de contração, obtida por eletromiografia de superfície, permite a obtenção de um índice de assimetria corrigido ${ }^{5,6,8,9}$.

Qualquer lesão em estruturas das vias do reflexo vestíbulo-cervical e vestíbulo-ocular pode resultar em alterações nos potenciais das respostas $^{9,10}$. Assim, alterações na mácula sacular ou do nervo vestibular inferior podem resultar em assimetria da amplitude ou ausência de resposta do lado acometido $^{7,8,9}$. O prolongamento das latências pode sugerir lesões retro labirínticas, especialmente no trato vestíbulo espinal ${ }^{9,10}$. O VEMP é um teste de rápida execução e pode ser usado para detecção precoce de alguns distúrbios vestibulares, como a doença de Ménière, em que o sáculo é uma das primeiras estruturas acometidas ${ }^{9,10}$.

Novas tecnologias na avaliação vestibular auxiliam o diagnóstico e subsidiam possíveis intervenções no paciente com tontura. Assim, torna-se necessário conhecer as principais características do VEMP cervical e ocular nas doenças vestibulares $^{9,10}$. O objetivo deste estudo foi analisar as respostas do VEMP cervical e ocular em indivíduos com doenças vestibulares e compará-las àquelas obtidas em indivíduos de mesma faixa etária e sexo, sem queixas de tontura pregressas e atuais.

\section{Método}

Os procedimentos desta pesquisa foram aprovados pelo Comitê de Ética da Universidade Federal de Minas Gerais (UFMG) sob o ${ }^{\circ}$ CAAE 56877316.1 .0000 .5149 (conforme Resolução 466/12 do Conselho Nacional de Saúde-CONEP). Trata-se de estudo observacional, transversal e analítico realizado no Observatório de Sáude Funcional em Fonoaudiologia da Faculdade de Medicina da UFMG. A casuística foi composta por 45 indivíduos com a idade entre 23 e 68 anos, sendo 27 
indivíduos do grupo controle (GC) e 18 do grupo com tontura (GT), pareados por sexo $(\mathrm{p}=0,232) \mathrm{e}$ idade $(\mathrm{p}=0,114)$.

Foram incluídos na pesquisa indivíduos maiores de 18 anos que concordaram voluntariamente em participar do estudo e assinaram o Termo de Consentimento Livre e Esclarecido. Foram excluídos pacientes com alteração de orelha externa à meatoscopia, história de cirurgia ou trauma otológico, dificuldade de rotação cervical autorrelatada e com algum distúrbio emocional importante autorrelatado. Trata-se de uma amostra de conveniência, composta por indivíduos da comunidade acadêmica.

O grupo com tontura foi constituído por participantes que estavam em acompanhamento no serviço de otorrinolaringologia do Serviço de Audiologia do Hospital das Clínicas da UFMG. Foram incluídos os indivíduos que possuíam diagnóstico otoneurológico estabelecido com base na análise do resultado do VEMP, v-HIT, prova calórica e da história clínica por um otoneurologista experiente, porém neste estudo limitou-se à análise dos dados do VEMP, apenas. Os indivíduos foram convidados a participar da pesquisa antes da realização do VEMP. O grupo controle foi composto por indivíduos sem história pregressa ou atual de tontura autorrelatada, pertencentes à comunidade acadêmica.

Inicialmente, o participante respondeu a um questionário que continha informações referentes ao passado otológico e à presença de possíveis alterações auditivas e vestibulares. Em seguida, realizou-se a meatoscopia e imitanciometria para descartar alterações de orelha média ${ }^{11}$. Para a realização do VEMP cervical e ocular utilizou-se o equipamento de potenciais evocados auditivos da marca Otometrics ${ }^{\circledR}$ modelo ICS Chartr EP 200, fones de inserção e eletrodos autoadesivos.

Para a realização do cVEMP, o participante permaneceu sentado em uma cadeira em local acusticamente tratado. Primeiramente, a pele foi limpa com pasta abrasiva. O eletrodo terra foi posicionado na fronte e os eletrodos ativos foram posicionados nos músculos esternocleidomastóideos direito e esquerdo. Os eletrodos de segundo canal foram posicionados abaixo dos ativos, direito e esquerdo para o registro da eletromiografia de superfície, e o eletrodo referência foi fixado na região do esterno. Foram utilizados fones de inserção para produção do estímulo tone burst por via aérea. Os partici- pantes foram orientados a realizar rotação lateral de cabeça para o lado oposto ao da orelha testada, com a finalidade de captar a resposta inibitória da contração muscular. Foram aceitas respostas obtidas com intensidade de contração muscular entre 50 e $200 \mu \mathrm{V}$. Foram aceitos valores de impedância abaixo de $5 \mathrm{kOhms}$. A intensidade inicialmente testada foi 95 dBNAn em $500 \mathrm{~Hz}^{4,5}$.

Para realização do oVEMP, o eletrodo ativo foi posicionado na região infraorbital contralateral ao lado testado, e o eletrodo de referência foi posicionado logo abaixo do eletrodo ativo. Foram utilizados fones de inserção para produção do estímulo tone burst por via aérea. Foram aceitas respostas obtidas com intensidade de contração muscular entre 50 e $200 \mu \mathrm{V}$. Foram aceitos valores de impedância abaixo de $5 \mathrm{kOhms}$. A intensidade inicialmente testada foi $95 \mathrm{dBNAn}$ em $500 \mathrm{~Hz}^{4,5}$. Os participantes foram orientados a permanecerem com a cabeça reta e olharem para cima, até o ponto de máximo alcance, durante a apresentação do estímulo. Para a análise dos valores de latência das ondas do cVEMP e oVEMP, considerou-se a literatura que define como alterados os valores de latência que superam 2,5 desvios padrões à normalidade sugerida ${ }^{4,5}$.

As variáveis analisadas no VEMP cervical foram as latências P13 e N23, amplitude, índice de assimetria e índice de assimetria corrigido. No oVEMP foram avaliadas as latências N10 e P15, amplitude e índice de assimetria. Após a coleta dos dados, estes foram tabulados em tabela de Excel e submetidos à análise estatística descritiva e inferencial por meio do programa Statistical Package for Social Sciences (SPSS) versão 20.0. A análise descritiva compreendeu medidas de tendência central (média e mediana), de dispersão (desvio padrão) e de posição (máximo e mínimo). A normalidade das amostras foi observada através do teste de Kolmogorov-Smirnov e Shapiro-Wilk.

A comparação entre grupos foi realizada por meio dos testes $\mathrm{t}$ de Student (para dados com distribuição normal) e Mann-Whitney (quando as variáveis não apresentaram distribuição normal). $\mathrm{O}$ teste Qui-quadrado foi aplicado para a comparação das frequências obtidas pelo cálculo do índice de assimetria, para comparação entre o sexo dos dois grupos e comparação entre os exames alterados, normais e ausentes dos dois grupos. Foi adotado o nível de significância de $5 \%(\mathrm{p} \leq 0,05)$ em todas as análises, intervalo de confiança de $95 \%$. 


\section{Resultado}

A média de idade da população estudada foi de $45,67( \pm 15,60)$ anos para o GC e $51,0( \pm 15,41)$ anos para o GT. O grupo GC apresentou-se com 7 (26\%) indivíduos do sexo masculino e 20 (74\%) indivíduos do sexo feminino, enquanto o grupo GT apresentou 2 (11\%) indivíduos do sexo masculino e $16(88 \%)$ indivíduos do sexo feminino. A amostra contou com um maior número de indivíduos do sexo feminino tanto no GT quanto no GC, mas não houve diferença estatisticamente significante entre os grupos para o sexo $(p=0,232)$ e idade $(p=0,114)$.

$\mathrm{Na}$ distribuição em porcentagem dos indivíduos por doenças, pode-se verificar que a Migrânea Vestibular esteve presente em 8 (44\%) dos indivíduos, seguida da Vertigem Posicional Paroxística Benigna (VPPB) em 4 (22\%), Neurite vestibular em 1 participante (6\%), Deiscência de canal superior em 2 (11\%), Doença de Ménière em 2 (11\%) e a Síndrome de Ramsay Hunt em 1 participante (6\%).
No exame VEMP cervical, as médias das latências de P13 e N23 e a amplitude das ondas não apresentaram diferença estatística, tanto na orelha direita quanto na orelha esquerda, na comparação entre os grupos. Os índices de assimetria e índices de assimetria corrigidos também foram iguais estatisticamente quando comparados entre os dois grupos (Tabela 1).

No exame oVEMP verificou-se que a latência de N10 do lado direito apresentou-se menor no grupo GT e a amplitude esquerda apresentou maiores valores no grupo GT quando comparado com o grupo GC. Os índices de assimetria não apresentaram diferença estatística entre os dois grupos (Tabela 2).

Quando comparadas as médias das amplitudes do oVEMP esquerdo, verificou-se que a Deiscência de canal semicircular superior apresentou valores maiores quando comparado às demais doenças vestibulares (Tabela 3 ).

Tabela 1. Comparação das medidas de tendência central, dispersão e posição da latência (ms), amplitude (uV), índice de assimetria (uV) e índice de assimetria corrigido (uV) para o VEMP cervical entre os grupos com e sem tontura.

\begin{tabular}{|c|c|c|c|c|}
\hline Parâmetros das ondas & Parâmetros analisados & Grupo Controle & Grupo com Tontura & p-valor* \\
\hline & Média & 15,30 & 14,56 & \\
\hline & Mediana & 15,17 & 14,42 & \\
\hline \multirow[t]{5}{*}{ P13E } & Desvio padrão & 1,76 & 1,65 & $* * 0,061$ \\
\hline & Mínimo & 11,00 & 9,33 & \\
\hline & Máximo & 20,00 & 17,67 & \\
\hline & Média & 24,24 & 23,33 & \\
\hline & Mediana & 24,00 & 23,50 & \\
\hline \multirow[t]{5}{*}{ N23E } & Desvio padrão & 2,51 & 2,23 & $* * 0,203$ \\
\hline & Mínimo & 19,33 & 17,67 & \\
\hline & Máximo & 29,17 & 28,33 & \\
\hline & Média & 135,91 & 164,73 & \\
\hline & Mediana & 119,14 & 136,20 & \\
\hline \multirow[t]{5}{*}{ Ampl.E.cerv } & Desvio padrão & 99,10 & 101,21 & $* 0,334$ \\
\hline & Mínimo & 17,82 & 29,15 & \\
\hline & Máximo & 445,74 & 388,60 & \\
\hline & Média & 29,28 & 26,35 & \\
\hline & Mediana & 27,01 & 27,05 & \\
\hline \multirow[t]{5}{*}{ IA.Corr } & Desvio padrão & 22,31 & 17,07 & $* 0,641$ \\
\hline & Mínimo & 0,56 & 4,96 & \\
\hline & Máximo & 80,59 & 55,16 & \\
\hline & Média & 22,43 & 24,64 & \\
\hline & Mediana & 19,39 & 19,88 & \\
\hline \multirow[t]{5}{*}{ IA.C } & Desvio padrão & 18,04 & 20,58 & $* * 0,708$ \\
\hline & Mínimo & 1,20 & 0,55 & \\
\hline & Máximo & 79,53 & 66,58 & \\
\hline & Média & 15,16 & 15,02 & \\
\hline & Mediana & 15,33 & 14,50 & \\
\hline \multirow[t]{3}{*}{ P13D } & Desvio padrão & 0,74 & 1,43 & $* * 0,191$ \\
\hline & Mínimo & 13,83 & 13,50 & \\
\hline & Máximo & 16,83 & 19,83 & \\
\hline
\end{tabular}




\begin{tabular}{|c|c|c|c|c|}
\hline Parâmetros das ondas & Parâmetros analisados & Grupo Controle & Grupo com Tontura & p-valor* \\
\hline \multirow{5}{*}{ N23D } & Média & 23,96 & 23,95 & \multirow{5}{*}{$* 0,996$} \\
\hline & Mediana & 23,58 & 23,83 & \\
\hline & Desvio padrão & 1,51 & 1,79 & \\
\hline & Mínimo & 21,50 & 21,33 & \\
\hline & Máximo & 26,50 & 28,33 & \\
\hline \multirow{5}{*}{ Ampl.D.cerv } & Média & 130,10 & 150,48 & \multirow{5}{*}{$* 0,466$} \\
\hline & Mediana & 107,79 & 110,02 & \\
\hline & Desvio padrão & 88,07 & 101,78 & \\
\hline & Mínimo & 30,01 & 39,84 & \\
\hline & Máximo & 396,77 & 419,91 & \\
\hline
\end{tabular}

*Teste $\mathrm{t} * *$ Teste Mann-Whitney

Legenda: Ampl.E.cerv = amplitude de onda esquerda VEMP cervical, IA.Corr = índice de assimetria corrigido, IA.c = índice de assimetria VEMP cervical, Ampl.D.cerv = amplitude de onda direita VEMP cervical.

Tabela 2. Comparação das medidas de tendência central, dispersão e posição da latência (ms), amplitude (uV) e índice de assimetria (uV) para o VEMP ocular entre os grupos com e sem tontura.

\begin{tabular}{|c|c|c|c|c|}
\hline Parâmetros das ondas & Parâmetros analisados & Grupo Controle & Grupo com tontura & p-valor* \\
\hline & Média & 14,63 & 14,33 & \\
\hline & Mediana & 14,25 & 14,38 & \\
\hline \multirow[t]{5}{*}{ P15E } & Desvio padrão & 1,34 & 1,64 & $* * 0,530$ \\
\hline & Mínimo & 12,67 & 10,67 & \\
\hline & Máximo & 17,33 & 16,50 & \\
\hline & Média & 10,95 & 10,25 & \\
\hline & Mediana & 10,63 & 9,92 & \\
\hline \multirow[t]{5}{*}{ N10E } & Desvio padrão & 1,20 & 1,22 & $* 0,054$ \\
\hline & Mínimo & 9,25 & 8,75 & \\
\hline & Máximo & 13,63 & 13,00 & \\
\hline & Média & 2,95 & 6,84 & \\
\hline & Mediana & 2,02 & 3,82 & \\
\hline \multirow[t]{5}{*}{ Ampl.E.oc } & Desvio padrão & 3,28 & 7,21 & $* 0,024$ \\
\hline & Mínimo & 0,32 & 0,70 & \\
\hline & Máximo & 15,67 & 26,48 & \\
\hline & Média & 38,25 & 27,42 & \\
\hline & Mediana & 32,62 & 19,55 & \\
\hline \multirow[t]{5}{*}{ IA.OC } & Desvio padrão & 24,26 & 22,19 & $* * 0,116$ \\
\hline & Mínimo & 2,27 & 7,73 & \\
\hline & Máximo & 78,42 & 92,93 & \\
\hline & Média & 15,23 & 14,76 & \\
\hline & Mediana & 14,88 & 14,63 & \\
\hline \multirow[t]{5}{*}{ P15D } & Desvio padrão & 1,21 & 1,65 & $* * 0,316$ \\
\hline & Mínimo & 13,25 & 12,33 & \\
\hline & Máximo & 17,33 & 17,17 & \\
\hline & Média & 10,94 & 10,03 & \\
\hline & Mediana & 10,59 & 9,84 & \\
\hline \multirow[t]{5}{*}{ N10D } & Desvio padrão & 1,38 & 0,91 & $* 0,018$ \\
\hline & Mínimo & 9,25 & 8,33 & \\
\hline & Máximo & 14,33 & 12,25 & \\
\hline & Média & 4,22 & 5,21 & \\
\hline & Mediana & 3,35 & 3,83 & \\
\hline \multirow[t]{3}{*}{ Ampl.D.oc } & Desvio padrão & 4,52 & 4,38 & $* 0,502$ \\
\hline & Mínimo & 0,26 & 0,44 & \\
\hline & Máximo & 21,25 & 14,22 & \\
\hline
\end{tabular}

*Teste $\mathrm{t} * *$ Teste Mann-Whitney

Legenda: Ampl.E.oc = amplitude de onda esquerda VEMP ocular, IA.oc = índice de assimetria ocular, VEMP ocular, Ampl.D.oc = amplitude de onda direita VEMP ocular. 
Tabela 3. Médias de amplitudes do VEMP ocular lado esquerdo entre as doenças vestibulares.

\begin{tabular}{lc}
\hline Doenças vestibulares & Amplitude VEMP ocular esquerdo \\
\hline Deiscência de canal superior & 14 \\
Doença de Ménière & 8,8 \\
Migrânea Vestibular & 6,2 \\
Vertigem Posicional Paroxistica Benigna & 6,1 \\
Neurite Vestibular & 1,7 \\
Síndorme de Ramsay Hunt & 1,5 \\
\hline
\end{tabular}

\section{Discussão}

Neste estudo verificou-se que no GT, a idade da população, em sua maioria, foi maior de 40 anos, o que concorda com a literatura que revela a alta prevalência de sinais e sintomas vestibulares em indivíduos nessa faixa etária, aumentando os sintomas com o avanço da idade ${ }^{3,5,6,7}$. Estes sinais e sintomas se manifestam na forma de tontura, perda auditiva, zumbido, alterações de equilíbrio corporal e quedas, sendo a tontura o sintoma mais comum após os 65 anos, com interferência direta na qualidade de vida ${ }^{3,6,12}$. Com o envelhecimento do sistema vestibular devido à perda da função das células ciliadas, ocorrem alterações nas respostas do VEMP cervical e ocular, como a diminuição da amplitude de resposta e aumento das latências e limiares. Essas alterações ocorrem devido ao envelhecimento da mácula sacular e das fibras musculares ${ }^{8,12}$.

O gênero predominante no GT foi o feminino, o que também concorda com a literatura que mostra a tontura como predominante no gênero feminino (1,3 para 1) e sua incidência aumenta em proporção direta com a idade, com pico entre 65 e $75 \operatorname{anos}^{3,6,9,12}$. No presente estudo, a doença vestibular mais frequente foi a Migrânea Vestibular, seguida da VPPB que é uma das mais frequentes afecções do sistema vestibular e prevalente no sexo feminino. Acredita-se que a variação hormonal mensal da mulher seja o gatilho para episódios de migrânea e de tonturas no período pré-menstrual, assim como a utilização de hormônios com finalidade contraceptiva que pode ser atribuída a fatores como a variação do ciclo hormonal ${ }^{3,13}$. A VPPB é a doença vestibular mais prevalente de acordo com a literatura, porém, por ser facilmente diagnosticada e tratada na clínica com manobras de reposicionamento de otólitos; estes indivíduos são tratados, na maioria das vezes, na atenção primária à saúde ${ }^{14,15}$.
A ausência de diferença estatística entre os resultados do VEMP quando comparado GT com o grupo GC se deve à heterogeneidade do grupo GT, constituído por indivíduos com diversas doenças otoneurológicas. Além disso, cada doença contou com um pequeno número de participantes, o que também pode ter interferido na ausência de diferença entre os grupos. Estes achados discordam da literatura que encontrou diferença entre respostas do VEMP na comparação entre indivíduos com e sem doenças vestibulares ${ }^{6,8,10}$.

No exame oVEMP verificou-se que o valor de amplitude à esquerda foi maior no GT, com significância estatística. Quando comparada a média desta amplitude com outras doenças, verificou-se que os indivíduos com deiscência de canal superior tiveram um resultado maior que as outras doenças, o que também pode ter influenciado no resultado. Este achado corrobora com a literatura que revela o aumento da amplitude das ondas com o aumento da intensidade do estímulo neste tipo de doença ${ }^{16,17}$. Este aumento se deve ao fato de que a deiscência de canal superior, ou "terceira janela", causa a diminuição da impedância da orelha interna, permitindo uma maior movimentação dos fluidos do labirinto membranoso e, consequentemente, permitindo uma maior deflexão dos sensores vestibulares a estímulos de pressão e som ${ }^{16,17}$.

A latência de N10 do lado direito apresentou-se menor no GT, quando comparado ao GC. Esse achado, que não corrobora com a literatura, pode ter surgido em virtude da pequena amostra deste estudo e da heterogeneidade do grupo GT, fato que foi também encontrado em outro estudo que comparou as respostas das orelhas em indivíduos sem queixas otoneurológicas 5 .

Dentre as limitações deste estudo cumpre destacar a variedade de doenças vestibulares, com um reduzido número de indivíduos com cada vestibulopatia. Este fato pode ter interferido na ausência de diferença estatística entre os grupos. Novos 
estudos com populações maiores são necessários para confrontar os achados deste estudo.

O presente estudo evidencia a contribuição do VEMP à bateria de exames otoneurológicos convencionais para o diagnóstico das variadas doenças vestibulares ${ }^{5,67,18}$. Sabe-se que o VEMP cervical e ocular são sensíveis às doenças vestibulares, confirmando a importância deste exame e sua aplicabilidade na prática clínica ${ }^{9,18,19}$.

O VEMP é um exame de fácil execução e interpretação, não invasivo, de relativo baixo custo, que não necessita nenhum preparo prévio à sua realização e não traz desconforto para o paciente, mas necessita de uma padronização para manter sua confiabilidade na rotina clínica ${ }^{18,19,20,21,22}$. A utilização de novas tecnologias em nossa prática clínica contribui para um diagnóstico mais detalhado das afecções vestibulares, possibilita um exame preciso e contribui para uma conduta clínica mais eficiente, com melhores resultados terapêuticos.

\section{Conclusão}

Não foram evidenciadas diferenças nas respostas do cVEMP entre os grupos neste estudo. Encontrou-se aumento da amplitude esquerda e o valor menor da latência N10 direita no grupo com tontura na análise do exame oVEMP. Acredita-se que a heterogeneidade de doenças otoneurológicas no grupo com tontura e o reduzido número de participantes tenham contribuído para esse desfecho.

\section{Referências}

1. Kleiner AFR, Schlitter DXC, Arias MDRS. The role of visual, vestibular, somatosensory and auditory systems for the postural control. Rev Neurocienc. 2011;19: 349-57.

2. Bisdorff A, Brevern MV, Lempert T, Newman-Toker DE. Classification of vestibular symptoms: Towards an international classification of vestibular disorders. J Vestib Res. 2009;19:113.

3. Oiticica J, Bittar RS. Population epidemiological study on the prevalence of dizziness in the city of São Paulo. Braz J Otorhinolaryngol. 2015; 81:167-761

4. Gonçalves DU, et al. Otoneurological evaluation: current good practice. Braz J Otorhinolaryngol. 2014; 80: 80-95

5. Silva TR, Resende LM, Santos MAR. Ocular and cervical vestibular evoked myogenic potential simultaneous in normal individuals. CoDAS. 2016; 28: 34-40.
6. Silva TR, Santos MAR, Resende LM, Labanca L, Caporali JFM, Sousa MR, Gonçalves DU. Applications of vestibularevoked myogenic potential: a systematic literature review. Audiol Commun Res. 2019; 24: e2037.

7. Silva BMP, Didoné DD, Sleifer P. Cervical vestibular evoked myogenic potentials in children and adolescents without vestibular complaints. Audiol Commun Res. 2017; 22: e1885.

8. Pereira AB, Silva GSM, Felipe L, Assunção ARM, Atherino CCT. Vestibular evoked myogenic potential (VEMP). Revista HUPE, Rio de Janeiro, 2015; 14: 56-59

9. Curthoys IS, et al. A review of mechanical and synaptic processes in otolith transduction of sound and vibration for clinical VEMP testing. J Neurophysiol. 2019;112: 259-276.

10. Felipe L, Santos MAR, Gonçalves DU. Vestibular evoked myogenic potential (Vemp): evaluation of responses in normal subjects. Pró-Fono. 2008; 20: 249-54.

11. Jerger J. Clinical experience with impedance audiometry. Arch Otolaryngol. 1970; 92: 311-24.

12. Ribeiro MBN, Morganti LOG, Mancini PC. Evaluation of the influence of aging on vestibular function by the video Head Impulse Test (v-HIT). Audiol Commun Res. 2019; 24: e2209.

13. Bittar RSM. Hormonal Labyrinth Diseases: Steroid Hormones, Estrogen and Progesterone. Int Arch Otorhinolaryngol. 1997;1(4): 32

14. Evren C, Demirbilek N, Elbistanli MS, Köktürk F, Çelik M. Diagnostic value of repeated Dix-Hallpike and roll maneuvers in benign paroxysmal positional vertigo. Braz J Otorhinolaryngol. 2017; 83: 243-8.

15. Messina A, Casani AP, Manfrin M, Guidetti G. Italian survey on benign paroxysmal positional vertigo. Acta Otorhinolaryngol Ital. 2017; 37: 328-335

16. Valente MCMB, Carnaúba ATL, Menezes PL, Cavalcanti MED. Cervical and Ocular VEMP Testing Diagnosing Superior Semicircular Canal Dehiscence. Distúrb Comum. 2017; 29: 613-614.

17. Camilo FFM, Gomes WRV, Lima CG, Vilella UPD, Siqueira LB. The Superior Semicircular Canal Dehiscence Syndrome. Revista Científica da FMC. 2018; 13: 2.

18. Silva TR, Resende LM, Santos MAR. Ocular vestibular evoked myogenic potential: literature review. Audiol Commun Res. 2016; 21: e1651.

19. Macambira YK, Carnaúba AT, Fernandes LC, Bueno NB, Menezes PL. Aging and wave-component latency delays in oVEMP and cVEMP: a systematic review with meta-analysis. Braz J Otorhinolaryngol. 2017; 83: 475-87.

20. Silva TR, de Resende LM, Santos MA. Combined ocular and cervical vestibular evoked myogenic potential in individuals with vestibular hyporeflexia and in patients with Ménière's disease. Braz J Otorhinolaryngol. 2017; 83: 330-40.

21. Rodriguez AL, et al. Optimization of Cervical and Ocular Vestibular Evoked Myogenic Potential Testing Using an Impulse Hammer in Adults, Adolescents, and Children. Otology \& Neurotology 2020; 41: 817-827.

22. Oya R, et al. Clinical significance of cervical and ocular vestibular evoked myogenic potentials in benign paroxysmal positional vertigo: a meta-analysis. European Archives of OtoRhino-Laryngology. 2019; 276: 3257-3265. 